\title{
The LOFAR Transients Key Science Project
}

\author{
Ben Stappers* ${ }^{\dagger}$ \\ Jodrell Bank Centre for Astrophysics, School of Physics and Astronomy, The University of \\ Manchester, Manchester M13 9PL. United Kingdom \\ E-mail: Ben.Stappers@manchester.ac.uk
}

\section{Rob Fender}

School of Physics and Astronomy, University of Southampton, Highfield, Southamption, S017

IBJ, $U K$

E-mail: rpfephys.soton.ac.uk

\section{Ralph Wijers}

Astronomical Institute 'Anton Pannekoek', University of Amsterdam, Kruislaan 403, 1098 SJ,

Amsterdam, the Netherlands

E-mail: Ralph.Wijers@uva.nl

The Transients Key Science Project (TKP) is one of six Key Science Projects of the next generation radio telescope LOFAR. Its aim is the study of transient and variable low-frequency radio sources with an extremely broad science case ranging from relativistic jet sources to pulsars, exoplanets, flare stars, radio bursts at cosmological distances, the identification of gravitational wave sources and even SETI. In these proceedings we will discuss some of the science goals of the TKP and with the roll out of the first few stations having begun we describe the current status of some of the pipelines being developed for the TKP.

Science and Technology of Long Baseline Real-Time Interferometry:

The 8th International e-VLBI Workshop, EXPReSO9

June 22 - 262009

Madrid, Spain

* Speaker.

†n behalf of the LOFAR Transients Key Science Project: http://www.transientskp.org/ 


\section{Introduction}

LOFAR is an extremely sensitive low-frequency radio telescope under construction in the Netherlands with long baselines extending throughout Europe (currently Germany, The United Kingdom, France and Sweden). It is the flagship project for ASTRON, and is the largest of the next generation radio telescopes, enroute to the global SKA project, that utilises large numbers of "cheap" receptors and vast computing resources. These receptors are arranged in groups of up to 96 called stations. Each station contains two types of receptors, called the low band antenna (LBA) which works in the frequency range $30-80 \mathrm{MHz}$, with limited sensitivity in the range $10-30 \mathrm{MHz}$, and the high band antenna (HBA) which works in the frequency range $110-240 \mathrm{MHz}(80-110 \mathrm{MHz}$ is deliberately avoided as it is dominated by FM radio transmissions). At the time of the meeting there are three stations in the field and many more are being rolled out. The end of 2009 will see 36 stations, in the core and longer baselines in the Netherlands, and a handful of European stations, in the field. Up to date information on the project can be found at http://www.lofar.org and for more technical details on LOFAR see the talk by Mike Garrett in these proceedings.

The science of LOFAR has been driven by six Key Science Projects: The Epoch of Reionization, Extra-galactic Surveys, Transients and pulsars, Cosmic Rays, Solar Physics and Cosmic Magnetism. In addition to these KSPs there will be an increasing fraction of open time available to the wider community. We will briefly discuss a subset of the science that the TKP will undertake and in particular discuss the Radio Sky Monitor. We'll also present a couple of highlights from the initial commissioning and pipeline development.

\section{The LOFAR Transients Key Science Project}

The LOFAR Transients Key Science Project (KSP) aims to study all variable and transient sources detected by LOFAR. Such sources can be broadly divided into two types:

Incoherent synchrotron emission: essentially all explosive events which inject energy into the ambient medium result in particle acceleration and/or compression/enhancement of magnetic fields, resulting in synchrotron emission. Such emission is likely to be initially self-absorbed at LOFAR frequencies, with a rise time corresponding to the timescale for the source to expand and become optically thin in a given band. This expansion timescale is proportional to the initial size of the emitting region divided by the expansion velocity. In approximate order of increasing rise time, sources which will be associated with such emission are jets from Cataclysmic Variables, X-ray binaries, Gamma-ray bursts (GRBs), Supernovae, and finally Active Galactic Nuclei (AGN). Under most conditions, synchrotron emission has a maximum brightness temperature of $\sim 10^{12} \mathrm{~K}$. An example of an outburst from a galactic binary system is given in Fig 1 below.

Note that many of the types of object responsible for explosive particle acceleration, e.g. Xray binaries, Gamma-ray bursts, are of intense interest to the high-energy astrophysics. LOFAR detections of such events, especially with the Radio Sky Monitor (see below), will undoubtedly therefore be used as triggers for optical / infrared / X-ray / gamma-ray follow-up (note for a typical X-ray binary outburst, the majority of the interesting X-ray behavior would still be observed even if the LOFAR trigger was $\sim 20$ days after the initial outburst due to the optical depth delay described above). Taken as an ensemble, observations of the synchrotron sources will provide a 

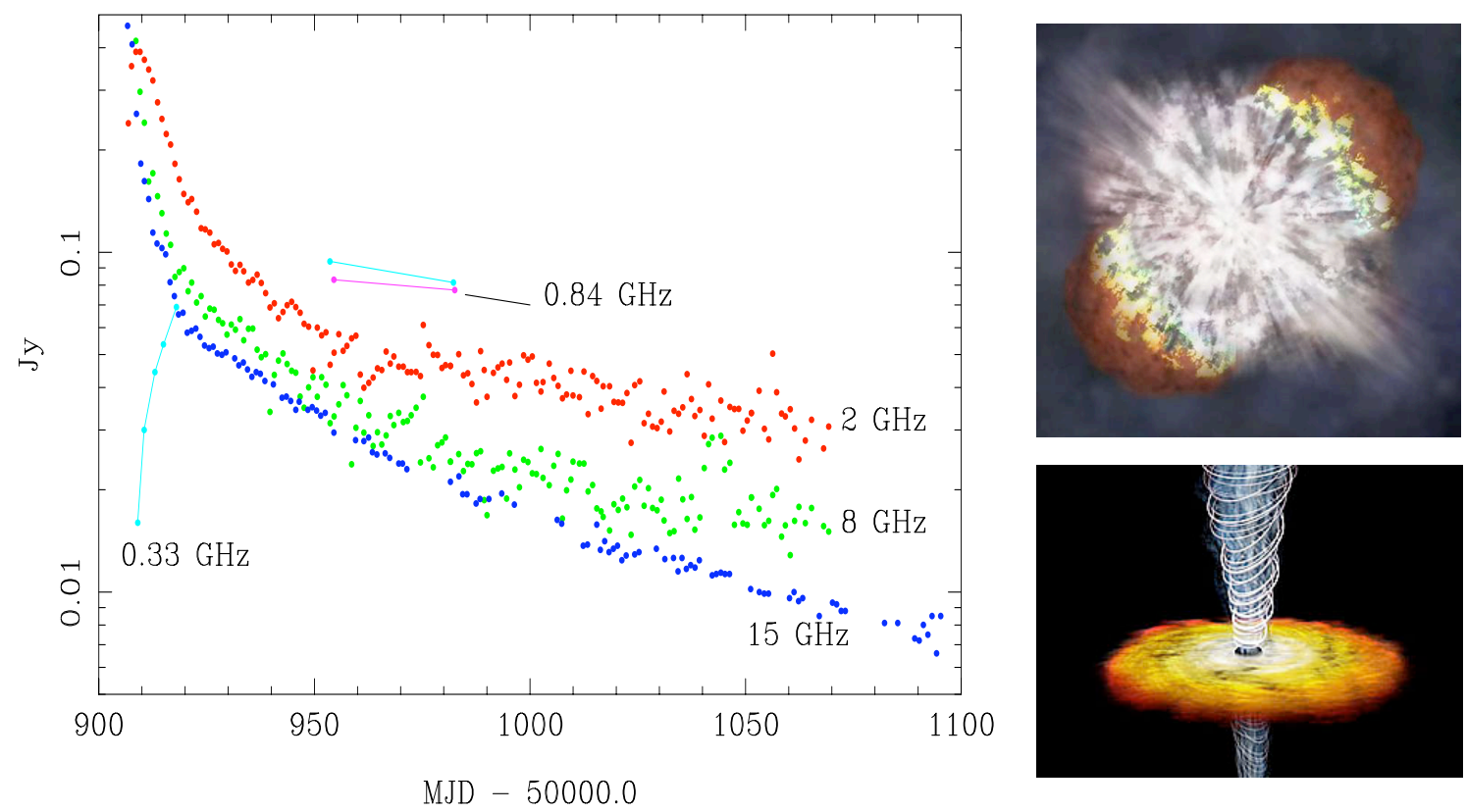

Figure 1: Evolution of a radio outburst from the X-ray transient CI Cam, with data from the Ryle Telescope $(15 \mathrm{GHz})$, the Green Bank Interferometer $(2$ and $8 \mathrm{GHz})$ and the Westerbork Synthesis Radio Telescope $(0.8$ and $0.3 \mathrm{GHz}$ ). The radio signal is due to synchrotron emission from an expanding source of accelerated electrons. By the time of the first radio observations ('externally triggered' by X-ray observations) the radio source is already optically thin at the highest frequency, $15 \mathrm{GHz}$. As the source expands, the flux density at different frequencies increases as the optical depth at that frequency decreases, but then subsequently decays once in the optically thin regime as expansion results in energy losses. The emission at $330 \mathrm{MHz}$, just above the LOFAR band, was detectable within a few days of the outburst, but did not peak until 20-30 days later. Such behaviour will be characteristic of explosive outburst events associated with e.g. relativistic jets, supernovae, GRB afterglows (as indicated by the cartoons to the right of the figure), with the rise and decay timescales being an increasing function of the luminosity of the event.

complete time-resolved census of particle acceleration in the local universe, shedding light on the energization of ambient media and sites of cosmic ray acceleration.

Coherent emission: several different types of radio emission with brightness temperatures $\geq 10^{12} \mathrm{~K}$, often resulting from very short durations, are lumped together under the title of 'coherent' radio emission. This is generally taken to mean groups of electrons moving together en masse. Examples include:

- Flare stars, brown dwarfs and active binaries are likely to be present in almost every LOFAR beam, giving off highly circularly-polarised radio bursts from coherent emission processes. Potential targets include M dwarf flare stars, active binaries like RS CVn and low mass L and T dwarfs.

- LOFAR will study radio emission from planets both within and beyond the Solar System. This includes imaging Jupiter's magnetosphere at high spatial and time resolution, imaging Jupiter's radiation belts, and studying planetary lightning from planets. We will also search for the radio bursts predicted from nearby so-called 'hot Jupiter' exoplanets. 


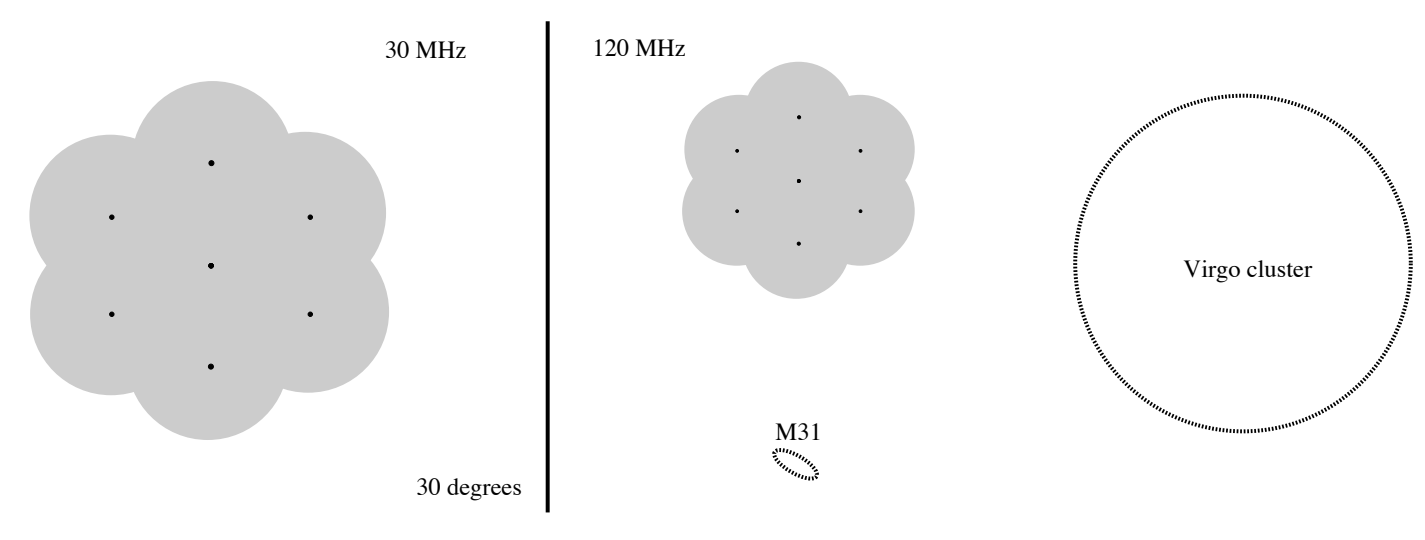

Northern galactic plane

Figure 2: An example of a Radio Sky Monitor (RSM) observing modes. In this example a hexagonal pattern is traced out using seven beams and an offset between pointing centres of FWHM $/ \sqrt{2}=6.4$ and 3.7 degrees at 30 and $120 \mathrm{MHz}$ respectively. The approximate angular size on the sky of M31, the Virgo cluster and the northern galactic plane are indicated for comparison. The angular resolution within these fields of view depends upon the longest baselines used - see Table 1 .

- LOFAR may detect extragalactic radio bursts, such as that reported by [5], to very large distances, possibly as far as $z \sim 7$. Probing the intergalactic medium and potentially being associated with gravitational wave sources and potential key sight lines for EOR studies.

In addition to all this, a major survey of classical radio pulsars will be undertaken, as well as the study of related objects such as Anomalous X-ray Pulsars (AXPs) and Rotating Radio Transients (RRATs). The LOFAR pulsar survey is expected to discover more than 1000 new pulsars, which may provide the majority of pulsars for the Pulsar Timing Array [1] in the northern hemisphere. Such a survey also has a fair chance of turning up the first pulsar - black hole binary. In addition, LOFAR will provide the sensitivity to allow us to study the individual pulses from an unprecedented number of pulsars including millisecond pulsars and the bandwidth and frequency agility to study them over a wide range which will provide vital new input for models of pulsar emission. This will provide us with an unparalleled survey of the population of massive star end-products within our galaxy.

Finally, beyond all this, LOFAR is almost guaranteed to produce many exciting physical discoveries simply because of the enormous range of parameter space it is exploring. This could include SETI, for which, depending on the nature of the signal, LOFAR may be the best hope prior to the SKA.

More information on the Transients KSP can be found at http://www.transientskp.org/.

\section{The Radio Sky Monitor}

The radio sky monitor (RSM) represents one of the four distinct approaches that will be used by the TKP to study the transient radio sky. We will also operate in phased array mode for very 
high time resolution studies (e.g. pulsars and planets); piggyback mode where we will attempt to search all LOFAR observations to look for variable sources; targeted mode will be used for monitoring known sources, or studies of more compact regions.

The RSM will optimally use the effective collecting area and field of view of LOFAR, which peaks in the HBAs and the LBAs at the low end of their respective frequency ranges. Figure 2 shows a comparison of the fields of view available to LOFAR and how that compares to various objects of interest. The RSM is likely to be used in three different modes:

- Rapid All-Sky Monitoring: Rapid shallow half-sky (hemispherical) surveys could be performed on short timescales in order to survey for rapid transients. In table $1, N_{2 \pi}$ corresponds to the number of beams required to tile out an entire hemisphere on the sky. This varies from of order 100 at $30 \mathrm{MHz}$ to $\sim 1600$ for $120 \mathrm{MHz}$ with international stations (which are larger and therefore have a smaller field of view). This is a very small number of pointings in order to survey half of the entire sky, and means that several minutes could be spent at each point in the sky (if such a scan were carried out over something like one day). This in turn means that $\sim$ mJy sensitivity (see $\sigma_{2 \pi}$ in table 1 ) surveys can be carried out daily.

- Zenith monitoring: Staring at the zenith optimises the sensitivity and beam stability of the telescope, whilst providing a sizeable and repeatedly monitored part of the sky. Using the patterns illustrated in Fig 5, mapping out the entire field of view which passes the zenith can be achieved in approximately 20 pointings at $30 \mathrm{MHz}$ and 30 at $120 \mathrm{MHz}$, tracking each field for about an hour, achieving (sub-)mJy sensitivity. In RA, DEC terms this delineates a strip at $54^{\circ}$ north of full width 26 degrees (i.e. extending from Dec +41 to +67 ).

- Galactic plane monitoring: Most of the northern galactic plane is visible from LOFAR and could be monitored for galactic transients. A large fraction of the 'known' radio transients, such as those associated with rotation-powered neutron stars and X-ray binary systems, are heavily concentrated towards the galactic plane (note that many sources e.g. flare stars, GRB afterglows, AGN are not). The RSM zenith-monitoring mode will sample the galactic plane between about $90 \leq l \leq 160$. However, much of the galactic plane will not be well sampled by this program, especially towards the galactic centre. In order to counterbalance this, we propose regular scans of the galactic plane. Since the galactic plane will also, however, suffer most from strong field sources and heavy dispersion at $30 \mathrm{MHz}$, these surveys will only be conducted at $120 \mathrm{MHz}$.

\section{Current Status}

Excellent progress is being made in developing and implementing the transients pipeline to detect transient sources. The aim is to be able to interrogate images of the sky made with logarithmically longer integration times starting from one second. On the shortest timescale, transients will be identified in these images within just a few seconds of the data being taken. We have developed an effective and efficient source detection algorithm which will extract source fluxes and positions and these will be compared in real time to a high speed relational database. Any new source, or a known source which has varied, will be immediately flagged and if it meets certain criteria a trigger will be raised. Triggers which meet yet further criterion will be passed on along 
to the community and will also be used to further trigger LOFAR for follow up observations allow for improved characterisation of the radio source.

We have carried out end-to-end testing of the initial versions of the pipeline, from simulated data which have resulted in successful triggers being generated. These pipelines are also being tested on data that was taken with the initial core station CS1 [2] and also on archival data from both the VLA and ATCA. In the processing of data from the VLA archive using an early version of the pipeline a "new" radio transient was found [3] and as part of the testing process we were able to raise a VOeventNet [4] trigger of the Liverpool telescope. These tests are essential for identifying bottlenecks and unexpected glitches in the pipeline.

We have also been carrying out extensive testing of the high time resolution modes of LOFAR. These have mainly been centered around observations of known pulsars up until now, but the mode will also be suitable for high time resolution observations of objects ranging from the Sun, to planets to known variable radio sources. Using only the 4 HBA tiles of CS1 we detected a handful of known radio pulsars as they transited. We have now progressed to being able to track known pulsars, in some cases for nearly three days, observe with individual sets of 24 tiles in the first stations rolled out. Most recently we have been able to observe with the coherently added combination of the two sets of 24 tiles which make up a core station. Development work is ongoing to allow for the formation of many so-called tied array beams, however it is already possible to form a handful of such beams, allowing for multiple look directions.

\section{Conclusions}

LOFAR is an exciting telescope for studying the variable radio sky. It will be able to detect sources of both incoherent synchrotron emission, such as GRBs and X-ray binaries, and coherent emission, such as planets, flare stars and radio emitting pulsars. The TKP will use dedicated pointing modes, all-sky surveys for pulsars and the unique RSM mode to study transients. The latter will come in three flavours, rapid all-sky monitoring, zenith monitoring and Galactic plane monitoring. The TKP has been developing a series of pipelines to find new sources including one to inspect large numbers of images which are currently being tested and developed and has been used to find a first transient in an archival dataset. The pulsar and tied-array mode pipeline is also being tested and developed and has already successfully been used to observe known sources. Towards the end of 2009 the first observations will be made of both pipelines in conjunction with the Million Source Shallow Survey which will make a shallow image of the entire Northern sky.

\section{References}

[1] Foster R.S., Backer D.C., 1990, ApJ, 361, 300

[2] Gunst A., van der Schaaf K., Bentum M.J., published in the Proceedings from SPS-DARTS 2006, The second annual IEEE BENELUX/DSP Valley Signal Processing Symposium, March 28-29 (2006), Antwerp, Belgium

[3] Bell M, et al. in prep 2009.

[4] www.voeventnet.org

[5] Lorimer D.R., Bailes M., McLaughlin M.A., Narkevic D.J., Crawford F., 2007, Science, 318, 777 